\title{
Análisis comparativo de la calidad percibida por espectadores en deportes de combate
}

\section{Comparative analysis of the perceived quality by combat sports spectators}

\author{
Angosto Sánchez, S., Martínez-Abellán, A., Rabadán Iniesta, J.C. y López-Gullón, J.M.
}

Facultad Ciencias del Deporte San Javier. Universidad de Murcia

\begin{abstract}
Resumen: El interés por conocer las percepciones del espectador respecto a la calidad y el valor del servicio, se ha trasladado a los eventos deportivos. Conocer cuáles son los elementos determinantes para alcanzar la satisfacción general del espectador, propiciará a los organizadores una mayor lealtad y mejora en la planificación de próximos eventos. Este estudio analiza, a través de un cuestionario, las distintas dimensiones de calidad percibida, que presenta un evento deportivo de combate, calidad de servicio global, satisfacción general, elementos de la competición e intenciones futuras, según la percepción del espectador en los Campeonatos de Espańa absolutos de las modalidades deportivas de Taekwondo y Lucha Olímpica. La dimensión de calidad percibida fue la peor valorada de forma global por los espectadores de los dos campeonatos, siendo la calidad de resultado la menos puntuada. Sin embargo, las intenciones futuras fueron el factor más valorado, existiendo pequeñas diferencias entre ambos eventos. Estos resultados son relevantes para los gestores de eventos deportivos para planificar estrategas para la mejora del servicio. Palabras clave: gestión deportiva, evento deportivo, deportes de combate, espectador, calidad percibida
\end{abstract}

Abstract: The interest in knowing the user's perceptions regarding the quality and value of the service has been transferred to the sports events. Knowing which are the determinant elements for the general satisfaction of the spectator, will cause to the organizations a greater loyalty and improvement in its sport projects. This study analyzes, through a questionnaire, the different dimensions from the quality perceived, services quality, competitions elements and futures intentions in the prediction of the general satisfaction of the spectator in Taekwondo and Wrestling Spanish Championships. The results indicated the quality dimension was the worst valued for the spectators in both sports, being the results quality the low punctuation dimension. However, the future intentions has got the best valued dimension for the spectators, there are no exist more differences between the both championships. These results would be relevant for managers in charge of sport events in order to plan strategies dedicated to services improvement.

Keywords: sport management, sporting event, combat sports, spectator, quality service

\section{Introducción}

Los deportes de combate pueden considerarse como unos de los más antiguos de los deportes que existen y uno de los deportes que acompaña al hombre desde épocas ancestrales (Seungmo, Greenwell, Andrew, Lee y Mahony, 2008).

Las modificaciones de los reglamentos oficiales de estos deportes, han propiciado que estos actualmente sean sustancialmente diferentes de los que se combatía a comienzos del Siglo XX (Ko, Kim, y Valacich, 2010). Estos cambios del reglamento han propiciado un mayor equilibrio del potencial físico entre rivales. Así mismo ha habido cambios que afectan no solo al competidor sino también al espectador como el color del equipamiento del luchador rojo y azul, la forma de puntuar de una manera más clara y con marcadores electrónicos, el video replay o challenger, las zonas y colores de combate etc.

La "gestión de eventos" se está convirtiendo en un campo profesional de rápido crecimiento en los últimos ańos en la que los turistas constituyen un mercado potencial para los eventos planificados (Getz, 2007), tanto los grandes eventos

Dirección para correspondencia [Correspodence address]: Salvador Angosto Sánchez. Facultad Ciencias del Deporte San Javier. Universidad de Murcia (España).

E-mail: angosto.salva@gmail.com de masas cómo Juegos Olímpicos o Campeonatos del Mundo, como los eventos menores que son aprovechados por la población para la planificación de sus vacaciones, dónde en la gran mayoría de ocasiones el número de participantes es mayor que el de espectadores y se realizan de forma anual (Gibson, Kaplanidou y Kang, 2012).

Los eventos deportivos de los deportes de combaten reúnen un público muy concreto. La asistencia de los espectadores a estos eventos depende en gran medida de la magnitud de los combates y cache de los contendientes. Por tanto, la calidad se está convirtiendo en una necesidad básica a la hora de elaborar estrategias (Gálvez y Morales-Sánchez, 2011), que permitan conocer la opinión de los espectadores y poder así diferenciarse dentro de un mercado de espectáculos deportivos altamente competitivo.

Shone y Bryan (2010, p. 4) realizan una definición de los eventos en general como "fenómenos que surgen de ocasiones no rutinarias y que tienen objetivos de ocio, culturales, personales $u$ organizativos establecidos de forma separada a la actividad normal diaria, cuya finalidad es ilustrar, celebrar, entretener $o$ retar la experiencia de un grupo de personas". 


\section{La Calidad de los Servicios}

En la actualidad dentro del ámbito empresarial, el concepto calidad es un tema crucial e importante. Cada vez más las empresas, tanto a nivel general como en el nivel deportivo, estudian ofrecer unos productos o servicios con la máxima calidad, con un fin común, poder llamar la atención de los futuros clientes o fidelizar los que la empresa tiene, sobre todo en la época que estamos viviendo, ya que es crucial para la supervivencia de la empresa.

Actualmente la calidad es bien necesario y básico que debe de tener la empresa a la hora de la elaboración de estrategias, con un fin principal, que es diferenciarse de las diferentes empresas del mercado, asegurando el progreso y estabilidad en el mercado tan competitivo que existe.

Dentro de los servicios, un aspecto importante que marca la diferencia con respecto a los productos, es que presentan características muy concretas, como la heterogeneidad, intangibilidad e inseparabilidad, ya que se produce y se consume en el momento y no tienen un propietario concreto (Afthinos, Theodorakis y Nassis, 2005; Chelladurai y Chang, 2000; Parasuraman, Zeithaml y Berry, 1985).

El gran interés por el conocimiento de cómo perciben los clientes el grado de calidad de los servicios deportivos, es una de las principales herramientas en las que las organizaciones, se apoyan y utilizan como una forma de profundizar en las características de los clientes o usuarios.

Según Calabuig, Molina y Núñez (2012), la calidad del servicio se podría definir como una evaluación a largo plazo post-consumo o post-uso, susceptible de cambio en cada transacción, fuente de desarrollo y cambio de las actitudes hacia el objeto de consumo o uso, y que es el resultado de procesos psicosociales de carácter cognitivo y afectivo.

En relación a la satisfacción, dentro de la literatura científica, se puede encontrar un acuerdo en el entendimiento del concepto satisfacción, que nos hablan de respuestas o evaluaciones relacionadas con el acto de compra. Encontrando también un consenso en la idea de que se trata de un juicio que varía en un continuo de lo insatisfecho a lo satisfecho (Crespo, 2011). En cuanto a la satisfacción del cliente, se reconoce como un constructo clave para los directivos y los investigadores interesado en las relaciones del servicio (Bodet, 2006).

Por tanto, el objetivo de este estudio es analizar y comparar la calidad percibida, las intenciones futuras y la satisfacción desde el punto de vista del espectador en el campeonato de Espańa absoluto de taekwondo y campeonato de Espańa Senior de Lucha Olímpica.

\section{Metodologia}

\section{Muestra}

La muestra estuvo conformada por un total de 82 espectadores, 44 de Taekwondo y 38 de Lucha. En el evento de Taekwondo el $54,5 \%$ fueron hombres y el $45,5 \%$ mujeres, con una media de edad de 37,33. Un 52,3\% tiene estudios universitarios, seguido de aquellos con estudios secundarios $(34,1 \%)$ y primarios (13,6\%). El 34,1\% afirma practicar cuatro o más veces actividad física por semana, seguido de dos o más veces por semana $(25,0 \%)$, tres veces por semana $(20,5 \%)$, ninguna vez $(11,4 \%)$ y por último una por semana $(9,1 \%)$. En cuanto a lo referente a la asistencia encontramos que la mayoría de los espectadores han acudido a otros eventos deportivos con anterioridad (75,0\%), seguido de eventos de combate y otros eventos $(15,9 \%)$, por primera vez a un evento $(6,8 \%)$ y por último a sólo eventos de combate $(2,3 \%)$.

En el evento de Lucha Olímpica el 54,1\% fueron hombres y el $45,9 \%$ mujeres, con una media de edad de 33,65. Un $48,6 \%$ tiene estudios secundarios, seguido de aquellos con estudios universitarios $(32,4 \%)$ y primarios $(18,9 \%)$. El 37,8\% afirma practicar cuatro o más veces actividad física por semana seguido de tres o más veces por semana $(24,3 \%)$, dos veces por semana $(16,2 \%)$, ninguna vez $(16,2 \%)$ y por último una por semana $(5,4 \%)$. En cuanto a lo referente a la asistencia encontramos que la mayoría de los espectadores han acudido a otros eventos deportivos con anterioridad $(78,4 \%)$, seguido de eventos de combate y otros eventos $(10,8 \%)$, por primera vez a un evento $(5,4 \%)$ y por último a sólo eventos de combate $(5,4 \%)$.

\section{Instrumento}

El cuestionario de calidad percibida en eventos deportivos por los espectadores de Pérez-Campos (2010) consta de diferentes áreas de evaluación diseñadas en función de la literatura existente. Las áreas con la que consta este instrumento son: datos sociodemográficos, calidad percibida, calidad de servicio global, satisfacción general, elementos de la competición e intenciones futuras.

\section{Propiedades psicométricas}

Para comprobar la validez del cuestionario propuesto, se aplicaron los tratamientos de fiabilidad y validez. La fiabilidad (cálculo de la consistencia interna de la escala), se determina a través del coeficiente alfa de Cronbach, obteniendo todos los factores índices adecuados en la mayoría de dimensiones (Tabla 1). 
Tabla 1. Fiabilidad de factores y escala.

\begin{tabular}{ccc}
\hline ESCALA & $\begin{array}{c}\text { Alfa de } \\
\text { Cronbach }\end{array}$ & $\begin{array}{c}\text { No } \\
\text { Ítems }\end{array}$ \\
\hline Calidad de personal & .947 & 6 \\
Calidad del entorno (tangibles) & .861 & 7 \\
Calidad del entorno (ambiente) & .839 & 4 \\
Calidad de resultados & .927 & 8 \\
Calidad percibida & .881 & 25 \\
Calidad servicio global & .954 & 5 \\
Satisfacción general & .943 & 5 \\
Satisfacción (elementos de la competición) & .626 & 3 \\
Intenciones futuras & .869 & 4 \\
\hline
\end{tabular}

El análisis de la validez del instrumento se llevó a cabo en la tesis doctoral de Pérez-Campos (2010) en la medición de la calidad percibida y satisfacción de espectadores y deportistas en el Campeonato de Espańa absoluto de Atletismo de pista cubierta y el Campeonato del Mundo de pista cubierta en Valencia.

\section{Procedimiento}

Los cuestionarios fueron administrados el día 15 de Febrero para el Campeonato de Espańa Senior de Taekwondo en el pabellón deportivo donde se celebró en Cartagena, y el día 14 de Marzo en Rivas-Vaciamadrid para el Campeonato de España Senior de Luchas Olímpicas.

Los cuestionarios se administraron durante los últimos momentos de la competición, entre la disputa de las finales y la entrega de trofeos, por voluntarios formados específicamente para el evento. Los voluntarios eran alumnos del Grado y Máster de Ciencias de la Actividad Física y el Deporte de la Universidad de Murcia.

\section{Análisis de datos}

El análisis de los datos se realizó, con el paquete estadístico SPPS 19 con licencia de la Universidad de Murcia. Las pruebas que se hicieron, fue análisis descriptivo y de frecuencias para la descripción de las variables, la prueba $t$ de Student para comprar la significación de resultados entre campeonatos, regresión lineal múltiple para la predicción de variables y el Alfa de Cronbach para calcular la fiabilidad del instrumento.

\section{Resultados y discusión}

Resultados de las dimensiones de calidad de los espectadores según el campeonato

Los resultados sobre las dimensiones de calidad de las escalas de calidad (Tabla 3) mostraron resultados para el campeonato de taekwondo una media de 5,76 para la calidad del entorno (ambiente), para la calidad de personal una puntuación de 5,35, seguido de la calidad del entorno (tangibles) y calidad de resultados con unas medias de 4,75 y 3,86 , respectivamente.

En cuanto al campeonato de luchas olímpicas, la dimensión más valorada fue calidad del entorno (ambiente) con una media de 6,20, seguido de calidad de personal 5,72, y calidad del entorno (tangibles) y calidad de resultados con unas medias de 5,55 y 4,52 en cada una de ellas.

Los resultados de ambos campeonatos arrojaron diferencias significativas entre ambos en las dimensiones de calidad del entorno (tangible), calidad del entorno (ambiente) y calidad de resultados, y una ligera tendencia a la significación en la calidad de personal.

Tabla 3. Resultados dimensiones calidad percibida.

\begin{tabular}{lccccc}
\hline & \multicolumn{2}{c}{ Taekwondo } & \multicolumn{2}{c}{ Lucha Olímpica } \\
\hline ESCALA & Media & DT & Media & DT & SIG \\
\hline Calidad de personal & 5,35 & 1,28 & 5,72 & 1,06 & .172 \\
Calidad del entorno (tangibles) & 4,75 & 1,16 & 5,55 & 1,02 & .001 \\
Calidad del entorno (ambiente) & 5,76 & 1,01 & 6,20 & 0,85 & .024 \\
Calidad de resultados & 3,86 & 1,15 & 4,52 & 0,86 & .004 \\
\hline
\end{tabular}

Comparando estos resultados con los obtenidos por Calabuig, Burillo, Crespo, Mundina, y Gallardo (2010); en las dimensiones de calidad de personal y calidad del entorno (tangibles) se aprecian puntuaciones medias más altas que las obtenidas en este estudio tanto en el evento de taekwondo como en el de lucha olímpica; 6,03 y 5,94, respectivamente. En la di- mensión de calidad del entorno (ambiente) se obtuvo medias bastante más altas en ambos eventos que las obtenidas por Hightower et al. (2002); que obtuvieron una media de 3,93. Observando las puntuaciones medias obtenidas en la dimensión de calidad de resultados con la de Crespo, Mundina, Calabuig, y Aranda (2011), existió una media muy similar 
a la obtenida en el evento de taekwondo, pero inferior en la obtenida en el evento de lucha olímpica. $(3,78)$. En lo referente a las dimensiones de calidad de personal $(5,27)$, calidad del entorno (tangibles) $(4,66)$ y calidad de personal $(5,27)$, se encontró con notas medias relativamente más bajas que las obtenidas en nuestro estudio en ambos eventos.

Resultados de otras dimensiones de los espectadores según el campeonato

Los resultados sobre las otras dimensiones de las escalas de calidad (Tabla 4) mostraron resultados para el campeonato de taekwondo una media de 6,34 para las intenciones futuras, para la satisfacción general una media de 5,54, para la satisfacción (elementos de competición) una media de 5,53, seguido de la calidad del servicio global y calidad percibida con medias de 5,11 y 4,93, respectivamente.

En cuanto al campeonato de luchas olímpicas, la dimensión con más puntuación fue intenciones futuras con una media de 6,39, seguido de satisfacción general con media de 6,13 , para la satisfacción (elementos de competición) una media de 5,96, y por último, calidad del servicio global y calidad percibida con medias de 5,55 y 4,52, respectivamente.

Los resultados de ambos campeonatos arrojaron diferencias significativas entre ambos en las dimensiones de calidad percibida, calidad del servicio global, satisfacción general e intenciones futuras, no encontrándose diferencias significativas en la dimensión de satisfacción (elementos de la competición).

Tabla 4. Resultados de las otras dimensiones.

\begin{tabular}{lccccc}
\hline & \multicolumn{2}{c}{ Taekwondo } & \multicolumn{2}{c}{ Lucha Olímpica } \\
\hline ESCALA & Media & DT & Media & DT & SIG \\
\hline Calidad percibida & 4,93 & 1.01 & 5,51 & 0,77 & .002 \\
Calidad servicio global & 5,11 & 1,25 & 5,89 & 1,10 & .009 \\
Satisfacción general & 5,54 & 1,10 & 6,13 & 0,88 & .028 \\
Satisfacción (elementos de la competición) & 5,53 & 0,92 & 5,96 & 0,81 & .752 \\
Intenciones futuras & 6,34 & 0,72 & 6,39 & 0,74 & .005 \\
\hline
\end{tabular}

Constatando estos datos con los obtenidos por Calabuig et al. (2010); en la dimensión de calidad percibida existió una media superior a las obtenidas en nuestro estudio en ambos eventos $(5,90)$. Sin embargo, en las dimensiones de satisfacción general $(6,12)$ y calidad de servicio global $(5,82)$ se encontraron medias superiores en el evento de taekwondo pero muy similares a los obtenidos en el evento de lucha olímpica.

En Hightower et al. (2002) la dimensión de calidad de servicio global reflejó una media de 5,32; siendo ésta superior a nuestra media en el evento de taekwondo pero inferior en el evento de lucha olímpica. En lo referente a la dimensión de intenciones futuras obtuvimos unas medias en los dos eventos muy similares a las obtenidas por Crespo et al. (2011) que muestra una media de 6,19. Sin embargo, se observaron diferencias más significativas en las dimensiones de calidad percibida $(4,80)$, satisfacción general $(5,47)$, y calidad del servicio global $(5,25)$, siendo medias más bajas que las obtenidas por nosotros en ambos eventos, a excepción de la dimensión de calidad de servicio global, que en el evento de taekwondo fue ligeramente superior.

\section{Análisis de regresión de los espectadores}

Predicción de la satisfacción de los espectadores en función de los factores de calidad

La validez del análisis del modelo regresión se realiza mediante la prueba ANOVA que se puede observar en la tabla 5 y tabla 6 , mostró que las variables independientes (los cuatro factores de calidad) que componen el modelo propuesto explican de forma significativa la variable dependiente (satisfacción general) de los espectadores del Campeonato de Espańa de Taekwondo y del Campeonato de España de Lucha Olímpica. Este estadístico fue ser el primer criterio para validar dicho modelo de regresión.

Tabla 5. Prueba ANOVA Cto. Taekwondo.

\begin{tabular}{ccccccc}
\hline Modelo & & Suma de cuadrados & Gl & Media cuadrática & F & Sig. \\
\hline \multirow{4}{*}{1} & Regresión & 32,397 & 4 & 8,099 & 15,706 &, $000^{c}$ \\
& Residual & 20,112 & 39 &, 516 & & \\
& Total & 52,509 & 43 & & & \\
\hline
\end{tabular}

Nota: a. CAMPEONATO = 1 b. Variable dependiente: SATISFACCION, c. Variables predictoras: (Constante), Personal, Tangibles, VAROOOO1, Ambiente 
Tabla 6. Prueba ANOVA Cto. Luchas Olímpicos.

\begin{tabular}{|c|c|c|c|c|c|c|}
\hline Modelo & & Suma de cuadrados & Gl & Media cuadrática & $\mathrm{F}$ & Sig. \\
\hline \multirow{3}{*}{1} & Regresión & 20,383 & 4 & 5,096 & 20,956 &, $000^{\circ}$ \\
\hline & Residual & 7,781 & 32 &, 243 & & \\
\hline & Total & 28,164 & 36 & & & \\
\hline
\end{tabular}

Con el fin de poder conocer que las variables independientes seleccionadas fueran buenos estimadores de la variable dependiente se empleó el estadístico del coeficiente de determinación (R2) y la prueba de Durbin-Watson. Mediante dichos estadísticos se expresa la relación que existe entre los factores de calidad y la satisfacción general de los espectadores de ambos eventos deportivos. Se pudo observar en la tabla (7 y 8), que el ajuste del modelo es bueno, con un $\mathrm{R}^{2}$ igual a 0,785 para el Campeonato de Espańa de Taekwondo, y un resulta- do mejor para el Campeonato de Espańa de Lucha Olímpica con $\mathrm{R}^{2}$ igual a 0,851 . Esto quiere decir que la variable satisfacción general se predice por las variables de calidad un 78.5\% en el caso del Cto. de Taekwondo y un $85.1 \%$ para el caso del Cto. de Luchas Olímpicas. Valores muy superiores a los obtenidos por Pérez-Campos (2010) en su tesis quien obtuvo una predicción del $49.9 \%$ para el Cto. de España y un pobre $37.9 \%$ para el Cto. del Mundo.

Tabla 7. Coeficientes de correlación del Cto. de Taekwondo.

\begin{tabular}{lccccc}
\hline Modelo & $\mathrm{R}$ & $\mathrm{R}$ cuadrado & $\mathrm{R}$ cuadrado corregida & Error típ. de la estimación & Durbin-Watson \\
\hline 1 &, $785^{\mathrm{b}}$ &, 617 &, 578 &, 7181138 & 2,225 \\
\hline
\end{tabular}

Tabla 8. Coeficientes de correlación del Cto. de Taekwondo.

\begin{tabular}{lccccc}
\hline Modelo & $\mathrm{R}$ & $\mathrm{R}$ cuadrado & $\mathrm{R}$ cuadrado corregida & Error típ. de la estimación & Durbin-Watson \\
\hline 1 &, $851^{\mathrm{b}}$ &, 724 &, 689 &, 4931167 & 2,017 \\
\hline
\end{tabular}

El resumen del modelo de análisis de regresión lineal múltiple de los factores de calidad podemos observarlo en la tabla (9 y 10), los coeficientes obtenidos por cada uno de los factores nos indican un nivel de significación $<0,05$ para las escalas de tangibles y personal para Taekwondo y las de ambiente, resultados y personal para las Luchas Olímpicas. De este análisis se deduce que durante el Campeonato de España de Taekwondo la variable que más información aporta para explicar la satisfacción de los usuarios es la calidad del entorno (tangibles). Por el contrario, en el caso del Campeonato de Espańa de Lucha Olímpica, la variable que más influye sobre la satisfacción de los usuarios durante el evento es la calidad del resultado.

Tabla 9. Coeficientes de regresión de los factores de calidad para el Cto. de Taekwondo.

\begin{tabular}{clccccc}
\hline \multirow{2}{*}{ Modelo } & & \multicolumn{2}{c}{ Coeficientes no estandarizados } & Coeficientes tipificados & \multirow{2}{*}{ t } & \multirow{2}{*}{ Sig. } \\
\cline { 3 - 5 } & & B & Error típ. & Beta & & \\
\hline \multirow{4}{*}{1} & (Constante) & 1,160 &, 639 & & 1,815 &, 077 \\
& Ambiente &, 231 &, 191 &, 212 & 1,213 &, 233 \\
& Resultados &,- 047 &, 147 &,- 049 &,- 321 &, 750 \\
& Tangibles &, 332 &, 154 &, 349 & 2,155 &, 037 \\
& Personal &, 309 &, 132 &, 360 & 2,344 &, 024 \\
\hline
\end{tabular}


Tabla 10. Coeficientes de regresión de los factores de calidad para el Cto. de Luchas Olímpicas.

\begin{tabular}{|c|c|c|c|c|c|c|}
\hline & \multirow{2}{*}{ Modelo } & \multicolumn{2}{|c|}{ Coeficientes no estandarizados } & \multirow{2}{*}{$\frac{\text { Coeficientes tipificados }}{\text { Beta }}$} & \multirow{2}{*}{$\mathrm{t}$} & \multirow{2}{*}{ Sig. } \\
\hline & & B & Error típ. & & & \\
\hline \multirow{5}{*}{1} & (Constante) & 967 & ,626 & & 1,545 & ,132 \\
\hline & Ambiente &, 347 & ,155 & ,334 & 2,242 & 032 \\
\hline & Resultados & ,455 &, 124 & ,448 & 3,685 & 001 \\
\hline & Tangibles &, 241 & ,118 & 279 & 2,039 &, 050 \\
\hline & Personal &,- 069 &, 100 &,- 083 &,- 689 & ,496 \\
\hline
\end{tabular}

\section{Conclusiones}

Las principales conclusiones que se obtuvieron de este trabajo fueron:

- La calidad del entorno (ambiente) fue la escala mejor valorada por los espectadores de Taekwondo como de Luchas Olímpicas, siendo las puntuaciones más altas en el segundo campeonato.

- La dimensión de calidad percibida peor valorada por los espectadores de ambos campeonatos fue la calidad de resultado.

- Las intenciones futuras fueron el factor más valorado en el resto de escalas tanto para los espectadores de Taekwondo como de Luchas Olímpicas, siendo éstas algo superiores respecto al otro campeonato.

- La dimensión de calidad percibida fue la peor valorada de forma global por los espectadores de Taekwondo y de Luchas Olímpicas.

- La satisfacción se predice a través de las variables de calidad percibida en un $78.5 \%$ para el Cto. de España de Taekwondo y un 85,1\% para el caso del Cto. de Espańa de Luchas Olímpicas.

\section{Aplicación práctica}

Este estudio tiene como objetivo poder observar las deficiencias en cuestión de calidad, que pueden surgir en un evento deportivo, en este caso, eventos de deportes de combate. Con la utilización de este instrumento tendremos una herramienta que nos pueda servir para mejorar la planificación, la gestión y la calidad de cualquier evento deportivo y poder conseguir un mayor número de espectadores y participantes. Y así poder crearles un sentimiento de lealtad hacia la prueba.

El uso de esta herramienta, permite obtener una información a corto plazo, que podrá ser utilizada para conocer y seleccionar los problemas existentes percibidos por los espectadores, pudiendo actuar sobre ellos, para aumentar el grado de satisfacción tanto de los espectadores como de los participantes en futuras ediciones demostrando, además, el compromiso de los organizadores hacia la calidad, y pudiendo así también optimizar los costes de la gestión de un evento deportivo y minimizarlos con una mejora en la planificación al estar totalmente orientada hacia el propio cliente.

\section{Referencias bibliográficas}

1. Afthinos, Y., Theodorakis, N. D., y Nassis, P. (2005). Customers' expectations of service in greek fitness centers. gender, age, type of sport center, and motivation differences. Managing Service Quality, 15(3), 245-258.

2. Añó, V. (2000). La Organización de grandes eventos deportivos. Arbor CLXV, 650 (Febrero 2000), 265-287.

3. Añó, V. (2003). La Organización y Gestión de Actividades Deportivas. Los Grandes Eventos. Barcelona: Inde.

4. Bodet, G. (2006). Investigating customer satisfaction in a health club context by an application of the tetraclasse model. European Sport Management Quarterly, 6(2), 149-165.

5. Bodet, G., y Bernache-Assollant, I. (2011). Consumer loyalty in sport spectatorship services: The relationships with consumer satisfaction and team identification. Psychology y Marketing, 28(8), 781-802.

6. Brady, K.M. \& Cronin, J.J. (2001). Some new thoughts on conceptualizing perceived service quality: A hierarchical approach. Journal of Marketing, 65 (July), 17-31.

7. Calabuig, F., Molina, N., Núñez, J. (2012). Una aplicación inicial del modelo tridimensional de calidad de servicio en centros deportivos privados. E-Balonmano: Revista de Ciencias del Deporte, 8(1), 67-81.
8. Calabuig, F.; Burillo, P.; Crespo, J.; Mundina, J.J. y Gallardo, L. (2010). Satisfacción, calidad y valor percibido en espectadores de atletismo. Revista Internacional de Medicina y Ciencias de la Actividad Física y el Deporte vol. 10 (40) pp. 577-593. Http://cdeporte.rediris.es/revista/ revista40/artsatisfaccion $182 . \mathrm{htm}$

9. Cerezuela, B. (2003). La información y documentación deportiva y los grandes eventos deportivos. Barcelona: Centro de Estudios Olímpicos, Universidad Autónoma de Barcelona.

10. Chelladurai, P., y Chang, K. (2000). Targets and standards of quality in sport services. Sport Management Review, 3(1), 1-22.

11. Clemes, M., Brush, G., y Collins, M. (2011). Analysing the professional sport experience: A hierchical approach. Sport Management Review, (in press).

12. Crespo, J. (2011). Los eventos deportivos: Calidad de servicio, valor percibido y satisfacción de los espectadores. Valencia: Universidad de Valencia.

13. Crespo, J., Calabuig, F., Mundina, J., Aranda, R. (2012). Valor percibido y satisfacción de los espectadores de eventos deportivos. En Calabuig, F., Molinos, M. y Sala, R. (Eds.), III Congreso Iberoamericano 
de Economía del Deporte. El deporte: Dinamizador económico (p. 47-50). Valencia, Espańa.

14. Gálvez, P. y Morales-Sánchez, V. (2011). Evaluación de la calidad en programas municipales deportivos: generalizabilidad y optimización de diseños de medida. Cuadernos de Psicología del Deporte, 11(2), 123-130.

15. Gálvez, P., Morales-Sánchez, V., Hernández-Mendo, A., Pérez-López, R. y López-Linares, A. (2012). Evaluación en gestión deportiva: calidad de servicios. Revista de Ciencias del Ejercicio FOD, 7(7), 29-37.

16. García-Fernández, J., Cepeda-Carrión, G. y Martín-Ruiz, D. (2012). La satisfacción de clientes y su relación con la percepción de calidad en Centro de Fitness: utilización de la escala CALIDFIT. Revista de Psicología del Deporte, 21(2), 309-319.

17. Getz, D. (2007). Event tourism: Definition, evolution, and research. Tourism management, 29(3), 403-428.

18. Gibson, H. J., Kaplanidou, K., y Kang, S. J. (2012). Small-scale event sport tourism: A case study in sustainable tourism. Sport management review, 15(2), 160-170

19. Grönroos, C. (1984). A service quality model and its marketing implications. European Journal of Marketing, 18(4)

20. Hightower, R., Brady, M. K. y Baker, T. L. (2002). Investigating the role of the physical environment in hedonic service consumption: An exploratory study of sporting events. Journal of Business Research, 55(9), 697-707.

21. Ko, Y.J., Kim, Y.K., y Valacich, J. (2010). Las artes marciales participación: la motivación. Consumer International Journal of Sports Marketing y Patrocinio, 11 (2), 105.
22. Luna-Arocas, R., y Mundina, J. (1998). El marketing estratégico del deporte: satisfacción, motivación y expectativas. Revista de Psicología del Deporte, 13, 169-174.

23. Mármol, A., Orquín Castrillón, F. J., y Sainz de Baranda, P. (2010). La infraestructura y el equipamiento, la prescripción del ejercicio y los servicios ofertados como índices de calidad de los centros fitness de Murcia. Cuadernos de Psicología del Deporte, 10(2), 85-91.

24. Mundina, J., Quintanilla, I., Sampedro, J., Calabuig, F. y Crespo, J. (2005). Estudio de la Calidad Percibida y la Satisfacción de los Espectadores y los Deportistas de los Juegos Mediterráneos Almería 2005. Valencia.

25. Parasuraman, A., Zeithaml, V. y Berry, L. (1985). A conceptual model of service quality and its implications for future research. Journal of Marketing, 49, 41-50.

26. Parasuraman, A., Zeithaml, V. y Berry, L. (1988). SERVQUAL: A multiple-item scale for measuring consumer perceptions of service quality. Journal of Retailing, 64(1), 12-40.

27. Pérez-Campos, C. (2010). Análisis de la calidad del servicio en los eventos deportivos. Calidad percibida y satisfacción de los espectadores y de los deportistas. Valencia: Universidad de Valencia.

28. Seungmo, K., Greenwell, TC, Andrew, DP, Lee, J., y Mahony, DF (2008). Un análisis de los motivos con espectadores en un deporte de combate individual: Un estudio de los fans de las artes marciales mixtas. Sport Marketing Trimestral, 17 (2), 109.

29. Shone, A. y Bryan, P. (2010). Successful event management: a practical handbook. London. 